\title{
The Synthesis of Blood Group Antigenic A Trisaccharide and Its Biotinylated Derivative
}

\author{
Ekaterina D. Kazakova (D, Dmitry V. Yashunsky and Nikolay E. Nifantiev *(D) \\ Laboratory of Glycoconjugate Chemistry, N. D. Zelinsky Institute of Organic Chemistry, Russian Academy \\ of Sciences, Leninsky pr. 47, 119991 Moscow, Russia; edkazakova@gmail.com (E.D.K.); \\ yashunsky1959@yandex.ru (D.V.Y.) \\ * Correspondence: nen@ioc.ac.ru
}

check for updates

Citation: Kazakova, E.D.; Yashunsky, D.V.; Nifantiev, N.E. The Synthesis of Blood Group Antigenic A Trisaccharide and Its Biotinylated Derivative. Molecules 2021, 26, 5887. https://doi.org/10.3390/molecules 26195887

Academic Editor: James Barker

Received: 26 August 2021

Accepted: 24 September 2021

Published: 28 September 2021

Publisher's Note: MDPI stays neutral with regard to jurisdictional claims in published maps and institutional affiliations.

Copyright: (c) 2021 by the authors. Licensee MDPI, Basel, Switzerland. This article is an open access article distributed under the terms and conditions of the Creative Commons Attribution (CC BY) license (https:/ / creativecommons.org/licenses/by/ $4.0 /)$.

\begin{abstract}
Blood group antigenic A trisaccharide represents the terminal residue of all A blood group antigens and plays a key role in blood cell recognition and blood group compatibility. Herein, we describe the synthesis of the spacered A trisaccharide by means of an assembly scheme that employs in its most complex step the recently proposed glycosyl donor of the 2-azido-2-deoxy-selenogalactoside type, bearing stereocontrolling 3-O-benzoyl and 4,6-O-(di-tert-butylsilylene)-protecting groups. Its application provided efficient and stereoselective formation of the required $\alpha$-glycosylation product, which was then deprotected and subjected to spacer biotinylation to give both target products, which are in demand for biochemical studies.
\end{abstract}

Keywords: blood group determinants; carbohydrates; stereoselective glycosylation

\section{Introduction}

Since the discovery of the ABO blood group system and the role of carbohydrate residues in blood antigens [1,2], there has been continued interest in developing new synthetic approaches to the assembly of carbohydrate blood group antigen determinants [3-10]. Besides playing an important role in blood cell recognition and blood group compatibility, blood transfusion, and organ transplantation [11-13], A trisaccharide and structurally related compounds can be used as haptens to test the carbohydrate specificities of plant [14] and mammalian lectins $[15,16]$ and serve as a model for conformational and spectral studies [17] of vicinally branched oligosaccharides. A trisaccharide derivatives can also serve as model compounds in the development of new biomedical technologies, since antibodies against this carbohydrate antigen are commercially available.

A trisaccharide represents the minimal terminal fragment of all blood group A antigens. It has a branched structure where the central $\beta-\mathrm{Gal}$ residue is glycosylated with $\alpha$-fucose at O-2 and with $\alpha$-galactosamine at O-3 (see Figure 1). Despite numerous works devoted to the synthesis of oligosaccharides related to blood group antigens, there are only a few papers dedicated specifically to the synthesis of A trisaccharide derivatives [4-6,9]. Herein, we report on the assembly of spacered A trisaccharide 1a and its biotinylated derivative $\mathbf{1 b}$, making use of the new galactose block 4 , which bears a set of convenient temporary protecting groups permitting selective liberation of HO-groups as well fucosyl donor 5 [18,19] and bicyclic 2-azido-2-deoxy-selenogalactoside 10 [20] containing stereocontrolling $\mathrm{O}$-protecting groups, which favor the required $\alpha$-(1,2-cis)-glycosylation. A bulky 4,6-O-(di-tert-butylsilylene)-protecting group at O-4 and O-6 was used to prevent the formation of undesirable $\beta$-glycosylation products [21] while a 3-O-benzoyl group was introduced to provide $\alpha$-stereocontrol through remote anchimeric participation [22].

Donor 10 was recently proposed $[20,23]$ and has been successfully applied in the syntheses of complex linear oligosaccharides related to bacterial and fungal antigenic polysaccharides (see a mini-review [23] and references therein). At the same time, there is still little published data on the glycosylation of vicinally branched oligosaccharides by 
means of 2-azido-2-deoxy-selenogalactoside donors as well as the application of the donors in the regioselective glycosylation of diolic acceptors. The studies discussed below were planned to fill these gaps.

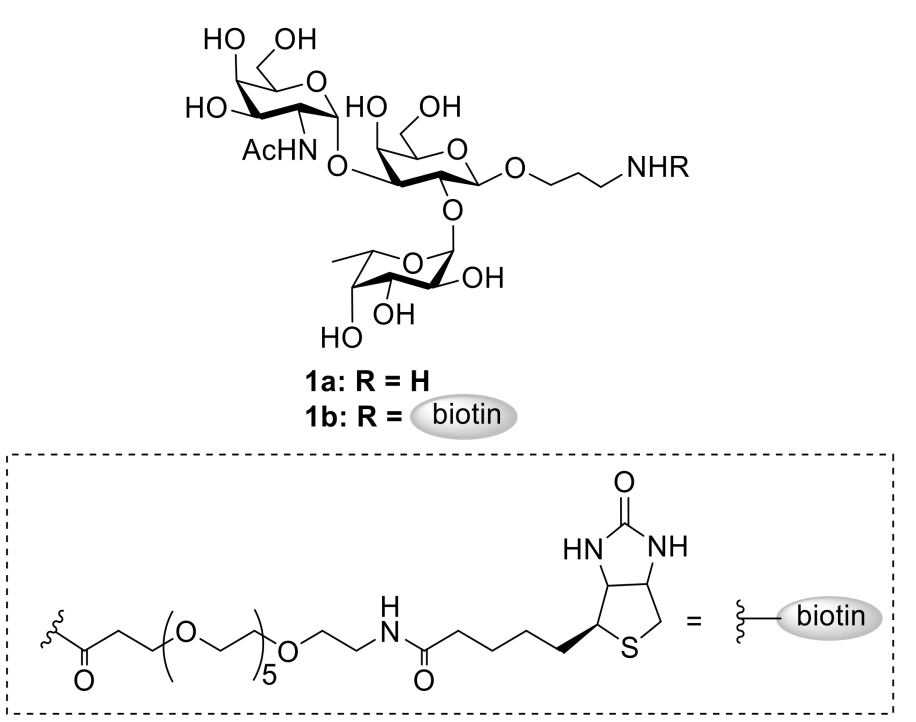

Figure 1. Target spacered A trisaccharide derivatives $\mathbf{1 a}$ and $\mathbf{1} \mathbf{b}$.

\section{Results and Discussion}

Key steps in the synthesis of spacered A trisaccharide 1a were the regio- and stereoselective building of three glycosidic bonds. While the formation of a $\beta$-glycoside bond is a straightforward task, $\alpha$-glycosylation by fucosyl and galactosamine donors requires the careful selection of protective groups and experimental conditions effective for $\alpha$-glycoside bond formation [24]. To promote the desired stereoselectivity, a new type of galactosyl acceptor 4 was easily synthesized from tetraol 2 [25] through the introduction of 3,4-Oisopropylidene and 6-O-benzoyl groups, which can be selectively removed in the presence of other protecting $\mathrm{O}$-substituents (Scheme 1).<smiles>OCC1(O)C(O)C(O)C(O)C(O)C1ON[NH+]F</smiles>

2
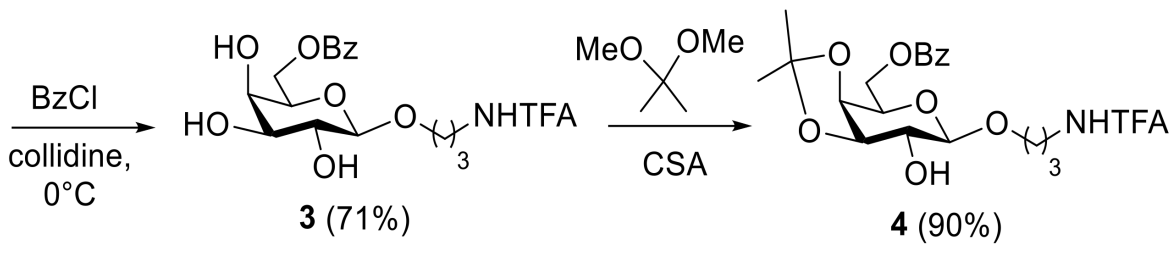

Scheme 1. Synthesis of galactosyl acceptor 4 .

To form the fucosyl block, donor 5 was used. This compound contains two benzoyl protecting groups at $\mathrm{O}-3$ and O-4, which, despite reducing the donor's activity, provide effective $\alpha$-directing glycosylation stereocontrol through remote anchimeric participation $[18,19]$. Fucosylation of galactoside 4 proceeded stereoselectively, giving an inseparable mixture of $\alpha$-isomer 6 and $\beta$ isomer 7 (Scheme 2) in the ratio 9:1 with a yield of $91 \%$. The anomeric configurations of the Fuc units in disaccharides 6 and 7 were confirmed by the characteristic values of the corresponding C-1 signals in ${ }^{13} \mathrm{C}$ NMR spectra and $J_{1,2}$ constants in the $1 \mathrm{H}$ NMR spectra (for 6: $95.5 \mathrm{ppm}$ and $3.4 \mathrm{~Hz}$; for 7: and $103.3 \mathrm{ppm}$ and $8.0 \mathrm{~Hz}$, respectively). The individual $\alpha$-isomer was purified after the removal of the $\mathrm{O}$ isopropylidene group, which gave the desired diol 8 in a 79\% yield. The value of coupling constant $J_{1,2}(3.6 \mathrm{~Hz})$ confirmed the $\alpha$-configuration of the Fuc unit in 8 . In addition to diol 8, its monohydroxy-derivative 9 was prepared by treatment with trimethyl orthobenzoate [26] followed by hydrolytic opening of the 3,4-O-orthobenzoic intermediate in an overall yield of $93 \%$ (Scheme 2). The presence of the 4-O-benzoyl group was confirmed by 
a downfield shift of the H-4Gal signal by 1.77 ppm between the spectra for compounds 8 and 9.

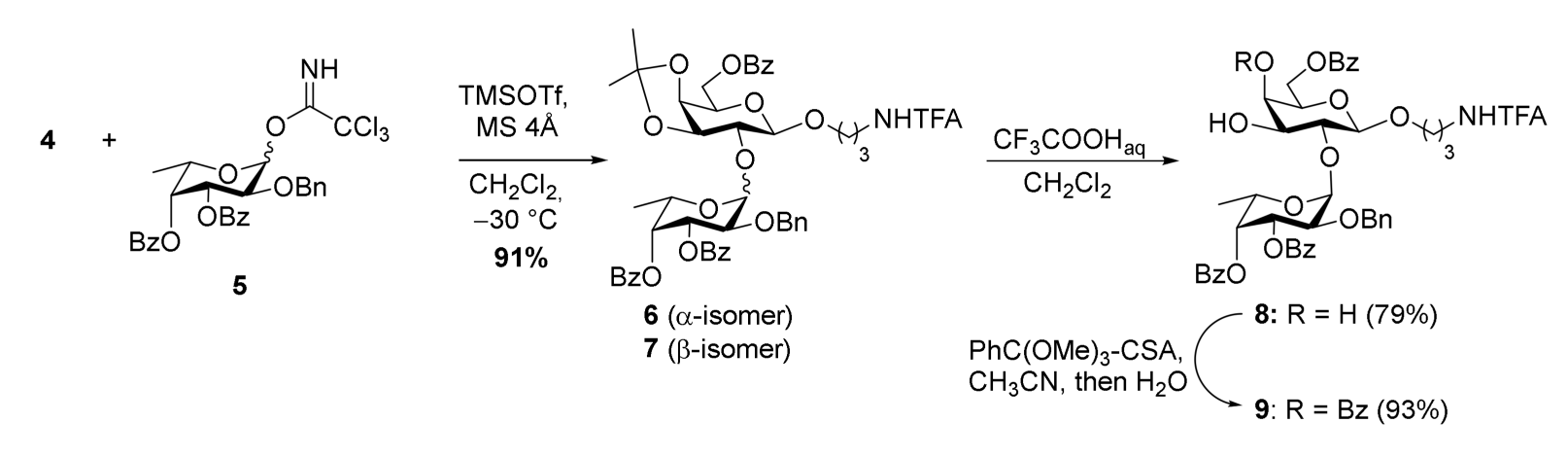

Scheme 2. Synthesis of disaccharides 8 and $\mathbf{9 .}$

The last step in the assembly of the trisaccharide A backbone was the glycosylation of disaccharide 8 with 2-azido-2-deoxy-galactosyl donor 10, which was prepared via azidophenylselenylation of a triacetylgalactal $[23,27]$ and subsequent selective protection. The glycosylation $\alpha$-stereoselectivity of donors of this type can be regulated by the reaction solvent [28] and specially selected types of O-protective groups [20-23,29,30].

It is known that equatorial hydroxyl groups are usually more reactive than axial ones [31,32]. Based on this assumption, we suggest that a regioselective 3-O-glycosylation of diol 8 would be possible. However, the reaction between disaccharide $\mathbf{8}$ and donor 10 yielded an inseparable mixture of products (Scheme 3). Presumably, it consisted of regioisomers $\mathbf{1 1}$ and $\mathbf{1 2}$ in a 2.8:1 ratio (NMR data), which were formed via $(1 \rightarrow 3)$ - and $(1 \rightarrow 4)$-glycosylation, respectively.

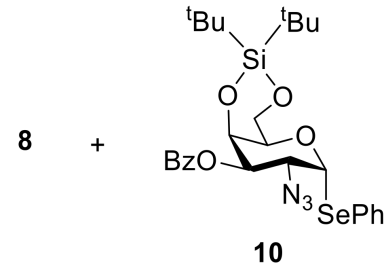

10

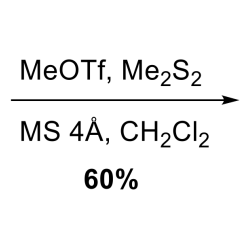

11: $\mathrm{R}^{1}=\mathrm{H} ; \mathrm{R}^{2}=$

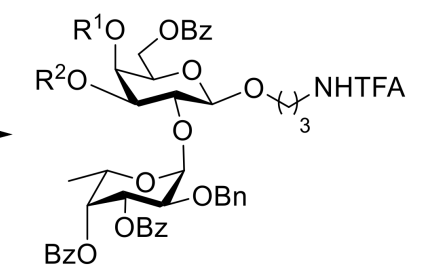

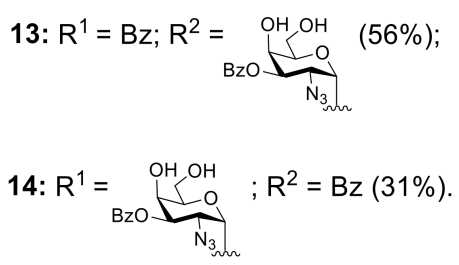

Scheme 3. Glycosylation of disaccharide 8 with donor 10.

To check our assumption, we treated the glycosylation products with $\mathrm{BzCl}$ in $\mathrm{Py}$ and then removed the 4,6-O-(di-tert-butylsilylene)-protection with HF/Py. As result, we obtained two separate compounds: $\alpha-3-\mathrm{O}$ - and $\alpha-4-\mathrm{O}-$ glycosylation products $\mathbf{1 3}$ and 14, which were identified by NMR spectroscopy. In particular, the formation of $\alpha$-glycoside bonds was confirmed by coupling constant $J_{1,2}$ for the GalN-unit $\left(3.8\right.$ and $3.6 \mathrm{~Hz}$ in ${ }^{1} \mathrm{H}$ NMR spectra for 13 and 14, respectively). Regioselectivity of the glycosylation reaction was confirmed by comparing the downfield signals $\mathrm{H}-3 \mathrm{Gal}$ and $\mathrm{H}-4 \mathrm{Gal}$ relative to each other in the ${ }^{1} \mathrm{H}$ NMR spectra (13: $4.24 \mathrm{ppm}$ for $\mathrm{H}-3 \mathrm{Gal}$; $5.99 \mathrm{ppm}$ for H-4Gal; 14: $5.42 \mathrm{ppm}$ for H-3Gal; 4.46 ppm for H-4Gal) and by downfield signals C-3Gal of 13 (73.2 ppm) and C-4Gal of 14 (74.5 ppm) in the ${ }^{13} \mathrm{C}$ NMR spectra.

As an alternative method to conduct 3-O-glycosylation with donor 10, we used monohydroxy-acceptor 9 (Scheme 4). As expected, the coupling of compounds 9 and 
10 was stereoselective and gave the desired trisaccharide 15 in an $81 \%$ yield, contaminated by traces of isomeric product that was formed due to the migration of a benzoyl group in 9 from O-4 to O-3 in the galactose unit during the reaction. Further removal of the di-tert-butylsilylene-group by HF/Py solution and chromatography purification gave the individual diol 13. The $\alpha$-configuration of the glycoside bond at the GalN-unit of $\mathbf{1 3}$ was confirmed by the characteristic value of the corresponding coupling constant $J_{1,2}(3.8 \mathrm{~Hz})$ in the ${ }^{1} \mathrm{H}$ NMR spectrum. Hydrogenolysis of $\mathbf{1 3}$ to remove the 2-O-benzyl group at the fucosyl residue and reduce the azide substituent to an amine and subsequent $\mathrm{N}$-acetylation resulted in the formation of trisaccharide $\mathbf{1 6}$ in an overall yield of $68 \%$. Its saponification gave the target spacered A trisaccharide 1a (73\%), which was then treated with the biotin derivative bearing an activated ester group $\mathbf{1 7}$ [33] to give the glycoconjugate $\mathbf{1 b}$.
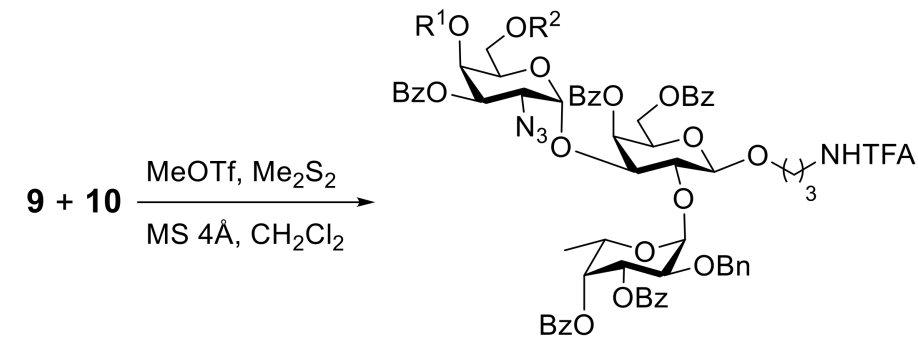

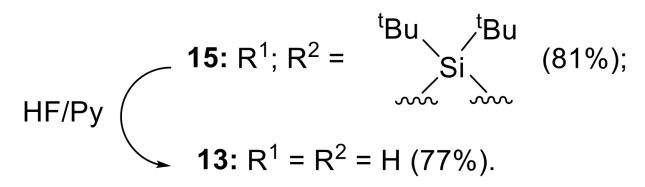

1. $\mathrm{Pd}(\mathrm{OH})_{2}, 1 \mathrm{M} \mathrm{HCl}$

$\mathrm{H}_{2}, \mathrm{MeOH}$, EtOAc

2. $\mathrm{Ac}_{2} \mathrm{O}, \mathrm{Et}_{3} \mathrm{~N}$

$\mathrm{CHCl}_{3}, \mathrm{MeOH}$

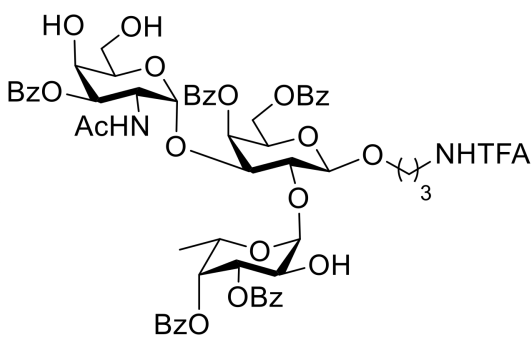

16 (68\%)

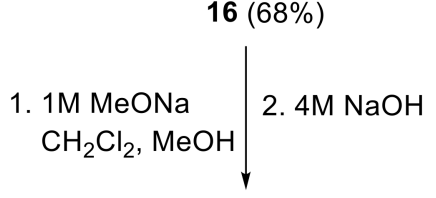

1a (73\%)

1b

(87\%)

$\mathrm{Et}_{3} \mathrm{~N}, \mathrm{DMF}$

Scheme 4. Assembly of the A trisaccharide backbone and preparation of target compound $\mathbf{1 a}$ and its biotinylated derivative $\mathbf{1} \mathbf{b}$.

\section{Materials and Methods}

\subsection{General Information}

All reagents were purchased at Sigma-Aldrich unless otherwise noted. MeCN and $\mathrm{CH}_{2} \mathrm{Cl}_{2}$ were distilled over $\mathrm{P}_{2} \mathrm{O}_{5}$ and $\mathrm{CaH}_{2}$. MeOH was distilled over $\mathrm{Mg}(\mathrm{OMe})_{2}$. Anhydrous pyridine and DMF were commercial (Sigma-Aldrich). Molecular sieves AW-300 MS ( $4 \AA$ ) were crushed and activated before reaction for $5 \mathrm{~min}$ at $400-500{ }^{\circ} \mathrm{C}$ in vacuo. Amberlite IR-120 (hydrogen form, Fluka) was washed with $1 \mathrm{M}$ aq. $\mathrm{HCl}, \mathrm{H}_{2} \mathrm{O}$, acetone, and dried.

Thin-layer chromatography (TLC) was carried out on aluminum sheets coated with silica gel 60 F254 (Merck). TLC plates were inspected under UV light $(\lambda=254 \mathrm{~nm})$ and developed with treatment by a mixture of $15 \% \mathrm{H}_{3} \mathrm{PO}_{4}$ and orcinol $(1.8 \mathrm{~g} / \mathrm{L})$ in EtOH-H $\mathrm{H}_{2} \mathrm{O}$ $(5: 95, v / v)$ followed by heating. Flash chromatography was performed on a Buchi Reveleris X2 system using Buchi FlashPure EcoFlex cartridges (irregular 40-63 $\mu \mathrm{m}$ silica). Column chromatography was performed with silica gel 60 (40-63 $\mu \mathrm{m}$, E. Merck). Gel-filtration was performed on a TSK-40 HW(S) column $(420 \times 25 \mathrm{~mm})$ and Sephadex G-15 column $(400 \times 17 \mathrm{~mm})$ by elution with $0.1 \mathrm{M} \mathrm{AcOH}$ in water at a flow rate of $0.5 \mathrm{~mL} \cdot \mathrm{min}^{-1}$ with a RI detector.

NMR spectra were recorded on Bruker Fourier 300HD (300 MHz), Bruker AV400 (400 MHz), or Bruker AV600 (600 MHz) spectrometers at temperatures denoted on the spectra. The resonance assignment in ${ }^{1} \mathrm{H}$ and ${ }^{13} \mathrm{C}$ NMR spectra was performed using 2D-experiments (COSY, HSQC). Chemical shifts are reported in ppm referenced to tetram- 
ethylsilane as a standard for $1 \mathrm{H}$ and solvent signal $\left(\delta=77.16\right.$ for $\left.\mathrm{CDCl}_{3}\right)$ for ${ }^{13} \mathrm{C} .{ }^{1} \mathrm{H}-\mathrm{NMR}$ spectra in $\mathrm{D}_{2} \mathrm{O}$ were registered with water suppression using a presaturation pulse sequence. See all NMR spectra in Supplementary Materials.

High-resolution mass spectra (HRMS) were recorded on a Bruker micrOTOF II instrument using electrospray ionization (ESI). The measurements were performed in positive ion mode (interface capillary voltage $-4500 \mathrm{~V})$ or in negative ion mode $(3200 \mathrm{~V})$; mass range from $m / z 50$ to $m / z 3000 \mathrm{Da}$; external or internal calibration was made with an electrospray calibrant solution (Fluka). A syringe injection was used for solutions in a mixture of acetonitrile and water $\left(50: 50 \mathrm{v} / \mathrm{v}\right.$, flow rate $\left.3 \mu \mathrm{L} \cdot \mathrm{min}^{-1}\right)$. Nitrogen was applied as a dry gas; interface temperature was set at $180^{\circ} \mathrm{C}$.

Glycosylation reactions were carried out in anhydrous solvent. Powdered molecular sieves were activated for $2 \mathrm{~h}$ at $180^{\circ} \mathrm{C}$ in vacuo using an oil pump before use in the reaction.

\subsection{Synthesis of Compounds $4,8,9,13,14,1 a$ and $1 b$}

3.2.1. 3-Trifluoroacetamidopropyl 3,4-O-Isopropylidene-6-O-Benzoyl- $\beta$-D-Galactopyranoside (4)

To a stirred solution of galactoside $2(312.5 \mathrm{mg}, 0.94 \mathrm{mmol})$ in collidine $(1.4 \mathrm{~mL}), \mathrm{BzCl}$ $\left(120 \mu \mathrm{L}, 1.03 \mathrm{mmol}, 1.1 \mathrm{eq}\right.$.) was added. The mixture was stirred at $0{ }^{\circ} \mathrm{C}$ for $2 \mathrm{~h}$, quenched with dimethylaminopropylamine (DMAPA), diluted with EtOAc, washed with $1 \mathrm{M}$ aq. $\mathrm{HCl}$, sat. aq. $\mathrm{NaHCO}_{3}$, the combined organic phase was dried by filtration through $\mathrm{Na}_{2} \mathrm{SO}_{4}$, and concentrated in vacuo. The residue was purified by flash chromatography $\left(\mathrm{CH}_{2} \mathrm{Cl}_{2}: \mathrm{MeOH}\right.$ $(0 \rightarrow 8 \%)$, giving $291.9 \mathrm{mg}(71 \%)$ of benzoylated monosaccharide 3 . It was dissolved on 2,2-dimethoxypropane $(5 \mathrm{~mL})$ and CSA $(73.5 \mathrm{mg})$ was added. The mixture was stirred at RT for $1.5 \mathrm{~h}$, quenched with $\mathrm{Et}_{3} \mathrm{~N}$, and co-evaporated with toluene in vacuo. The residue was purified by flash chromatography (toluene:ethyl acetate $20 \rightarrow 50 \%)$, giving $286.8 \mathrm{mg}(90 \%$; summary yield $64 \%)$ of monosaccharide $4 .[\alpha]_{D}{ }^{16}+29.5\left(c 1, \mathrm{CHCl}_{3}\right) .{ }^{1} \mathrm{H}$ NMR $(400 \mathrm{MHz}$, $\mathrm{CDCl}_{3}$ ): 1.36 and 1.53 (both s, on $\left.3 \mathrm{H}, 2 \mathrm{CH}_{3}\right), 1.82-1.91\left(\mathrm{~m}, 2 \mathrm{H}, \mathrm{OCH}_{2} \mathrm{CH}_{2} \mathrm{CH}_{2} \mathrm{~N}\right), 2.44$ $(\mathrm{s}, 1 \mathrm{H}, \mathrm{OH}), 3.49\left(\mathrm{qd}, 2 \mathrm{H}, J=2.7 \mathrm{~Hz}, 6.1 \mathrm{~Hz} ; \mathrm{OCH}_{2} \mathrm{CH}_{2} \mathrm{CH}_{2} \mathrm{~N}\right), 3.57(\mathrm{t}, \overline{1 \mathrm{H}}, J=7.7 \mathrm{~Hz}$; $\mathrm{H}-2$ ), 3.68-3.76, and 3.92-3.98 (both $\mathrm{m}$, on $1 \mathrm{H}, \mathrm{OCH}_{2} \mathrm{CH}_{2} \mathrm{CH}_{2} \mathrm{~N}$ ), 4.09-4.13 (m, $1 \mathrm{H}, \mathrm{H}-3$ ), 4.13-4.18 (m, 1H, H-5), 4.21-4.24 (m, 2H, H-1, H-4), 4.60-4.63 (m, 2H, H-6), 7.42-8.05 (m, $5 \mathrm{H}, \mathrm{Ph}) .{ }^{13} \mathrm{C}$ NMR $\left(100 \mathrm{MHz}, \mathrm{CDCl}_{3}\right): 26.3,28.0\left(2 \mathrm{CH}_{3}\right), 28.3\left(\mathrm{OCH}_{2} \mathrm{CH}_{2} \mathrm{CH}_{2} \mathrm{~N}\right), 38.3$ $\left(\mathrm{OCH}_{2} \mathrm{CH}_{2} \underline{\mathrm{C}} \mathrm{H}_{2} \mathrm{~N}\right), 63.6(\mathrm{C}-6), 69.0\left(\mathrm{OCL}_{2} \mathrm{CH}_{2} \mathrm{CH}_{2} \mathrm{~N}\right), 71.4(\mathrm{C}-5), 73.3(\mathrm{C}-4), 73.5(\mathrm{C}-2), 79.0$ (C-3), 102.4 (C-1), 110.7 ( $\underline{\mathrm{C} M e} 2), 128.5,129.6,133.3,166.3(\mathrm{Ph}), 166.3$ (ㄷOPh). HRMS (ESI) $m / z$ : found $\left[\mathrm{M}+\mathrm{NH}_{4}\right]^{+} 495.1941, \mathrm{C}_{21} \mathrm{H}_{26} \mathrm{~F}_{3} \mathrm{NO}_{8}$ calcd for $\left[\mathrm{M}+\mathrm{NH}_{4}\right]^{+} 495.1949$.

3.2.2. 3-Trifluoroacetamidopropyl 2-O-Benzyl-3,4-Di-O-Benzoyl- $\alpha$-L-Fucopyranosyl$(1 \rightarrow 2)-6-O-B e n z o y l-\beta-D-G a l a c t o p y r a n o s i d e ~(8)$

A carefully dried mixture of donor $5(389.8 \mathrm{mg}, 0.64 \mathrm{mmol}, 1.45 \mathrm{eq}$.$) and galactosyl ac-$ ceptor 4 (211.6 mg, $0.44 \mathrm{mmol}, 1$ eq.) was dissolved in $\mathrm{CH}_{2} \mathrm{Cl}_{2}(6 \mathrm{~mL})$ and molecular sieves $4 \AA$ ( $600 \mathrm{mg})$ were added. The mixture was cooled to $-30^{\circ} \mathrm{C}$ and TMSOTf $(8 \mu \mathrm{L}, 44 \mu \mathrm{mol}$, 0.1 eq.) was added. The mixture was stirred and warmed up to ambient temperature for $3 \mathrm{~h}$ and then quenched with one drop of $\mathrm{Et}_{3} \mathrm{~N}$. The mixture was filtered through a Celite pad with $\mathrm{CH}_{2} \mathrm{Cl}_{2}$, and the filtrate was evaporated in vacuo. The residue was purified by flash chromatography (toluene:ethyl acetate $0 \rightarrow 10 \%$ ), giving $369.2 \mathrm{mg}(91 \%)$ of disaccharide 6 with inseparable minor quantity ( 11\%) of $\beta$-isomer $7 .{ }^{1} \mathrm{H}$ NMR $\left(600 \mathrm{MHz}, \mathrm{CDCl}_{3}\right.$, inter alia): $4.38(\mathrm{~d}, 1 \mathrm{H}, J=8.0 \mathrm{~Hz} ; \mathrm{H}-1 \mathrm{Gal}$ of 7$), 4.43(\mathrm{~d}, 1 \mathrm{H}, J=8.2 \mathrm{~Hz} ; \mathrm{H}-1 \mathrm{Gal}$ of 6$), 4.99(\mathrm{~d}$, $1 \mathrm{H}, J_{1,2}=8.02 \mathrm{~Hz} ; \mathrm{H}-1 \mathrm{Fuc}$ of 7$), 5.63(\mathrm{~d}, 1 \mathrm{H}, J=3.4 \mathrm{~Hz} ; \mathrm{H}-1$ Fuc of 6$) .{ }^{13} \mathrm{C} \mathrm{NMR}(150 \mathrm{MHz}$, $\mathrm{CDCl}_{3}$, inter alia): 95.5 (C-1Fuc of 6), 101.3 (H-1Gal of 6), 102.7 (H-1Gal of 7), and 103.3 (C-1Fuc of 7).

To the disaccharide mixture (369.2 $\mathrm{mg}, 0.4 \mathrm{mmol})$ in $\mathrm{CH}_{2} \mathrm{Cl}_{2}(4 \mathrm{~mL})$ was added $90 \%$ aq. TFA $(400 \mu \mathrm{L})$. After $15 \mathrm{~min}$, the mixture was diluted with $\mathrm{CH}_{2} \mathrm{Cl}_{2}$, washed with sat. aq. $\mathrm{NaHCO}_{3}$, the organic phase was dried by filtration through $\mathrm{Na}_{2} \mathrm{SO}_{4}$ and concentrated in vacuo. The residue was purified by flash chromatography $\left(\mathrm{CHCl}_{3}\right.$ : acetone $\left.0 \rightarrow 10 \%\right)$, giving $278 \mathrm{mg}(79 \%$; summary yield $72 \%)$ of disaccharide 8. [ $\alpha]_{D}{ }^{17}-95.1\left(\right.$ c $\left.1, \mathrm{CHCl}_{3}\right) .{ }^{1} \mathrm{H}$ NMR $\left(600 \mathrm{MHz}, \mathrm{CDCl}_{3}\right): 1.18\left(\mathrm{~d}, 3 \mathrm{H}, J=6.5 \mathrm{~Hz} ; \mathrm{CH}_{3}-\mathrm{Fuc}\right), 1.83-1.97\left(\mathrm{~m}, 2 \mathrm{H}, \mathrm{OCH}_{2} \mathrm{CH}_{2} \mathrm{CH}_{2} \mathrm{~N}\right)$, 
2.73 (s, 1H, OH-4Gal), 3.39-3.46, and 3.53-3.60 (both m, on $1 \mathrm{H}, \mathrm{OCH}_{2} \mathrm{CH}_{2} \mathrm{CH}_{2} \mathrm{~N}$ ), 3.64-3.73 (m, 2H, OC $\left.\underline{\mathrm{H} H C H} \mathrm{CH}_{2} \mathrm{~N}, \mathrm{H}-2 \mathrm{Gal}\right), 3.76(\mathrm{dd}, 1 \mathrm{H}, \mathrm{J}=3.0 ; 9.0 \mathrm{~Hz} ; \mathrm{H}-3 \mathrm{Gal}), 3.86(\mathrm{t}, 1 \mathrm{H}$, $J=6.4 \mathrm{~Hz} ; \mathrm{H}-5 \mathrm{Gal}), 3.99-4.05$ (m, 2H, OCH$\left.\underline{H} \mathrm{CH}_{2} \mathrm{CH}_{2} \mathrm{~N}, \mathrm{H}-4 \mathrm{Gal}\right), 4.18$ (dd, 1H, J = 3.5; $10.3 \mathrm{~Hz}$; H-2Fuc), 4.33 (s, 1H, OH-3Gal), 4.38 (d, 1H, J = 7.8 Hz; H-1Gal), 4.50 (q, 1H, $J=6.6 \mathrm{~Hz}$; H-5Fuc), 4.59-4.62 (m, 1H, H-6Gal), 4.64 and 4.72 (AB system, $J=11.4 \mathrm{~Hz}$; $\left.\mathrm{CH}_{2}-\mathrm{Ph}\right), 5.16(\mathrm{~d}, 1 \mathrm{H}, \mathrm{J}=3.6 \mathrm{~Hz} ; \mathrm{H}-1 \mathrm{Fuc}), 5.65$ (d, 1H, J = 3.3 Hz; H-4Fuc), 5.74 (dd, 1H, $J=3.2 ; 10.3 \mathrm{~Hz} ; \mathrm{H}-3 \mathrm{Fuc}), 7.24-7.64$ (m, 20H, $4 \mathrm{Ph}) .{ }^{13} \mathrm{C}$ NMR (150 MHz, $\left.\mathrm{CDCl}_{3}\right): 16.1$ (C-6Fuc), $28.4\left(\mathrm{OCH}_{2} \mathrm{CH}_{2} \mathrm{CH}_{2} \mathrm{~N}\right), 38.6\left(\mathrm{OCH}_{2} \mathrm{CH}_{2} \mathrm{CH}_{2} \mathrm{~N}\right), 63.0(\mathrm{C}-6 \mathrm{Gal}), 66.1$ (C-5Fuc), 67.9 (C-4Gal), $68.8\left(\mathrm{OCH}_{2} \mathrm{CH}_{2} \mathrm{CH}_{2} \mathrm{~N}\right), 71.0$ (C-3Fuc), 72.0 (C-4Fuc), 72.3 (C-5Gal), 73.1 (C-3Gal), 74.2 ( $\left.\mathrm{CH}_{2}-\mathrm{Ph}\right), 74.3$ (C-2Fuc), 81.9 (C-2Gal), 100.6 (C-1Fuc), 102.0 (C-1Gal), 128.3-136.3 (4 Ph), $157.1\left(\mathrm{COCF}_{3}\right), 165.7,165.8,166.3$ (3 $\left.\mathrm{COPh}\right)$. HRMS (ESI) $m / z$ : found $[\mathrm{M}+\mathrm{Na}]^{+}$ 904.2764, $\mathrm{C}_{45} \mathrm{H}_{46} \mathrm{~F}_{3} \mathrm{NO}_{14}$ calcd for $[\mathrm{M}+\mathrm{Na}]^{+} 904.2763$.

\subsubsection{3-Trifluoroacetamidopropyl 2-O-Benzyl-3,4-di-O-Benzoyl- $\alpha$-L-Fucopyranosyl-

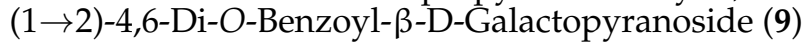

To a stirred solution of disaccharide $8(278 \mathrm{mg}, 0.32 \mathrm{mmol})$ in $\mathrm{CH}_{3} \mathrm{CN}(3 \mathrm{~mL})$ was added trimethyl orthobenzoate $(181 \mu \mathrm{L}, 1.06 \mathrm{mmol}, 3.3 \mathrm{eq}$.$) and catalytic amounts of$ CSA $(15 \mathrm{mg})$ up to $\mathrm{pH}<7$. At the end of the reaction according to TLC, $\mathrm{H}_{2} \mathrm{O}(50 \mu \mathrm{L})$ was added. The mixture was diluted with $\mathrm{CH}_{2} \mathrm{Cl}_{2}$, washed with sat. aq. $\mathrm{NaHCO}_{3}$, the organic phase was dried by filtration through $\mathrm{Na}_{2} \mathrm{SO}_{4}$ and concentrated in vacuo. The residue was purified by flash chromatography (toluene:ethyl acetate $0 \rightarrow 20 \%$ ), giving $294.3 \mathrm{mg}$ (93\%) of disaccharide 9. $[\alpha]_{D}{ }^{28}-92.3$ (c 1, $\left.\mathrm{CHCl}_{3}\right) .{ }^{1} \mathrm{H} \mathrm{NMR}\left(400 \mathrm{MHz}, \mathrm{CDCl}_{3}\right)$ : 1.19 (d, 3H, $\left.J=6.5 \mathrm{~Hz} ; \mathrm{CH}_{3}-\mathrm{Fuc}\right), 1.91-2.00\left(\mathrm{~m}, 2 \mathrm{H}, \mathrm{OCH}_{2} \mathrm{CH}_{2} \mathrm{CH}_{2} \mathrm{~N}\right), 3.49-3.57$ (m, 2H, $\left.\mathrm{OCH}_{2} \mathrm{CH}_{2} \mathrm{CH}_{2} \mathrm{~N}\right), 3.71-3.77$ (m, 1H, H-2Gal), 3.80-3.87 (m, $\left.\overline{\mathrm{H}}, \mathrm{OC} \underline{\mathrm{H}} \mathrm{HCH}_{2} \mathrm{CH}_{2} \mathrm{~N}\right), 4.01-$ 4.09 (m, 2H, $\left.\overline{\mathrm{OC}} \underline{H}_{\mathrm{HCH}} \mathrm{CH}_{2} \mathrm{~N}, \mathrm{H}-3 \mathrm{Gal}\right), 4.10-4.17$ (m, 2H, H-5Gal $\left.\mathrm{H}-2 \mathrm{Fuc}\right), 4.40$ (dd, 1H, $J=5.8,11.28 \mathrm{~Hz}$; H-6aGal), 4.49-4.67 (m, 5H, H-5Fuc, H-6bGal, H-1Gal, CH $-\mathrm{Ph}), 5.10$ (d, $1 \mathrm{H}, J=3.6 \mathrm{~Hz}$; H-1Fuc), 5.66 (d, 1H, J = 3.3 Hz; H-4Fuc), 5.73 (dd, 1H, J= 3.2; $10.4 \mathrm{~Hz}$; $\mathrm{H}-3 \mathrm{Fuc}), 5.77$ (d, 1H, J = 3.4 Hz; H-4Gal), 7.03-8.18 (m, 25H, $5 \mathrm{Ph}) .{ }^{13} \mathrm{C} \mathrm{NMR}(100 \mathrm{MHz}$, $\left.\mathrm{CDCl}_{3}\right): 16.1$ (C-6Fuc), $28.3\left(\mathrm{OCH}_{2} \mathrm{CH}_{2} \mathrm{CH}_{2} \mathrm{~N}\right), 39.0\left(\mathrm{OCH}_{2} \mathrm{CH}_{2} \mathrm{CH}_{2} \mathrm{~N}\right), 62.3(\mathrm{C}-6 \mathrm{Gal}), 65.9$ (C-5Fuc), 69.5 (C-4Gal), $69.7\left(\mathrm{OCH}_{2} \mathrm{CH}_{2} \mathrm{CH}_{2} \mathrm{~N}\right), 71.0$ (C-3Fuc), 71.73 (C-5Gal), 72.0 (C-4Fuc), 72.5 (C-3Gal), 73.5 (C-2Fuc), 73.9 ( $\left.\underline{\mathrm{CH}}_{2}-\mathrm{Ph}\right), 81.7$ (C-2Gal), 100.8 (C-1Fuc), 102.3 (C-1Gal),

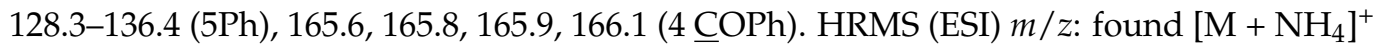
1003.3472, $\mathrm{C}_{52} \mathrm{H}_{50} \mathrm{~F}_{3} \mathrm{NO}_{15}$ calcd for $\left[\mathrm{M}+\mathrm{NH}_{4}\right]^{+} 1003.3471$.

3.2.4. 3-Trifluoroacetamidopropyl 2-Azido-3-O-Benzoyl-2-Deoxy- $\alpha$-D-Galactopyranosyl$(1 \rightarrow 3)$-[2-O-Benzyl-3,4-Di-O-Benzoyl- $\alpha$-L-Fucopyranosyl-( $\rightarrow 2)$ ]-4,6-Di-O-Benzoyl- $\beta$-DGalactopyranoside (13) and 3-Trifluoroacetamidopropyl 2-Azido-3-O-Benzoyl-2-Deoxy$\alpha$-D-Galactopyranosyl-(1 $\rightarrow 4)$-[2-O-Benzyl-3,4-Di-O-Benzoyl- $\alpha$-L-Fucopyranosyl- $(1 \rightarrow 2)]$ 4,6-Di-O-Benzoyl- $\beta$-D-Galactopyranoside (14)

A: A carefully dried mixture of donor $\mathbf{1 0}(29.4 \mathrm{mg}, 50 \mu \mathrm{mol}, 1.1 \mathrm{eq}$.$) and disaccharide$ acceptor 8 (39.7 $\mathrm{mg}, 45 \mu \mathrm{mol})$ was dissolved in $\mathrm{CH}_{2} \mathrm{Cl}_{2}(1 \mathrm{~mL})$ and molecular sieves 4 $\AA$ (100 mg) were added. After $10 \mathrm{~min}$, MeOTf $(27 \mu \mathrm{L}, 0.25 \mathrm{mmol}, 5.5$ eq. $)$ and $\mathrm{Me}_{2} \mathrm{~S}_{2}$ ( $22 \mu \mathrm{L}, 0.25 \mathrm{mmol}, 7.5$ eq.) were added. The mixture was stirred for $2 \mathrm{~h}$ at room temperature and quenched with one drop of $\mathrm{Et}_{3} \mathrm{~N}$. The mixture was filtered through a Celite pad with $\mathrm{CH}_{2} \mathrm{Cl}_{2}$ and the filtrate was washed with sat. aq. $\mathrm{NaHCO}_{3}$. The organic phase was separated, and the solvent was evaporated in vacuo. The residue was purified by flash chromatography (toluene:ethyl acetate $0 \rightarrow 10 \%$ ), giving $35.5 \mathrm{mg}$ of an inseparable mixture of trisaccharides $\mathbf{1 1}$ and 12. To a solution of purified mixture in Py $(0.3 \mathrm{~mL})$ was added $\mathrm{BzCl}(7 \mu \mathrm{L}, 54 \mu \mathrm{mol}, 2$ eq.) while stirring. After $30 \mathrm{~min}$, the mixture was diluted with EtOAc, washed with $1 \mathrm{M}$ aq. $\mathrm{HCl}$, sat. aq. $\mathrm{NaHCO}_{3}$, the combined organic phase was dried by filtration through $\mathrm{Na}_{2} \mathrm{SO}_{4}$, and concentrated in vacuo. The residue was purified by flash chromatography (toluene:ethyl acetate $0 \rightarrow 10 \%$ ). To a solution of the obtained compound in Py $(0.5 \mathrm{~mL})$ was added $40 \%$ aq. HF $(83 \mu \mathrm{L})$. After $10 \mathrm{~min}$, the mixture was diluted with EtOAc, washed with $1 \mathrm{M}$ aq. $\mathrm{HCl}$, sat. aq. $\mathrm{NaHCO}_{3}$, the combined organic phase was dried by filtration through $\mathrm{Na}_{2} \mathrm{SO}_{4}$ and concentrated in vacuo. The residue was 
purified by flash chromatography (toluene: ethyl acetate $20 \rightarrow 50 \%$ ), giving $19.3 \mathrm{mg}(34 \%)$ of trisaccharide 13 and $10.3 \mathrm{mg}$ (19\%; purity $85 \%)$ of trisaccharide 14. 13: $[\alpha]_{D}{ }^{20}+10.4(c$ 1, $\left.\mathrm{CHCl}_{3}\right) .{ }^{1} \mathrm{H}$ NMR $\left(400 \mathrm{MHz}, \mathrm{CDCl}_{3}\right): 1.21\left(\mathrm{~d}, 3 \mathrm{H}, J=6.4 \mathrm{~Hz} ; \mathrm{CH}_{3}-\mathrm{Fuc}\right), 1.92-1.99(\mathrm{~m}$, $\left.2 \mathrm{H}, \mathrm{OCH}_{2} \mathrm{CH}_{2} \mathrm{CH}_{2} \mathrm{~N}\right), 3.41-3.60\left(\mathrm{~m}, 2 \mathrm{H}, \mathrm{OCH}_{2} \mathrm{CH}_{2} \mathrm{CH}_{2} \mathrm{~N}\right), 3.72-3.85(\mathrm{~m}, 3 \mathrm{H}, \mathrm{H}-6 \mathrm{GaalN}$, OCH $\left.\mathrm{HCH}_{2} \mathrm{CH}_{2} \mathrm{~N}\right), 3.89(\mathrm{dd}, 1 \mathrm{H}, J=3.7 ; 10.9 \mathrm{~Hz}, \mathrm{H}-2 \mathrm{GalN}), 4.01-4.09(\mathrm{~m}, 2 \mathrm{H}, \mathrm{H}-2 \mathrm{Gal}$, $\left.\mathrm{OCH}{ }^{-} \mathrm{CH}_{2} \mathrm{CH}_{2} \mathrm{~N}\right), 4.10-4.19$ (m, 2H, H-5Gal, H-2Fuc), 4.24 (dd, $\left.1 \mathrm{H}, J=3.4 ; 9.6 \mathrm{~Hz}, \mathrm{H}-3 \mathrm{Gal}\right)$, $4.32(\mathrm{~d}, 1 \mathrm{H}, J=2.8 \mathrm{~Hz} ; \mathrm{H}-4 \mathrm{GalN}), 4.35-4.41$ (m, 2H, H-5GalN, H-6aGal), 4.55-4.65 (m, 3H, H-1Gal, H-5Fuc, H-6bGal), 4.72-4.82 (m, 2H, CH2-Ph), 5.26 (d, 1H, J = 3.7 Hz, H-1Fuc), $5.44(\mathrm{dd}, 1 \mathrm{H}, J=2.9 ; 10.9 \mathrm{~Hz} ; \mathrm{H}-3 \mathrm{GalN}), 5.54(\mathrm{~d}, 1 \mathrm{H}, J=3.8 \mathrm{~Hz}, \mathrm{H}-1 \mathrm{GalN}), 5.61-5.67(\mathrm{~m}$, 2H, H-4Fuc, H-3Fuc), 5.99 (d, 1H, J = 3.4 Hz, H-4Gal), 7.05-8.20 (m, 30H, 6Ph). ${ }^{13} \mathrm{C}$ NMR $\left(100 \mathrm{MHz}, \mathrm{CDCl}_{3}\right)$ : 16.2 (C-6Fuc), $28.5\left(\mathrm{OCH}_{2} \mathrm{CH}_{2} \mathrm{CH}_{2} \mathrm{~N}\right), 38.4\left(\mathrm{OCH}_{2} \mathrm{CH}_{2} \mathrm{CH}_{2} \mathrm{~N}\right), 57.3(\mathrm{C}-$ 2GalN), 62.17 (C-6Gal), 62.9 (C-6GalN), 64.6 (C-4Gal), 65.6 (C-5Fuc), 68.52 (C-4GalN), 69.1 $\left(\mathrm{OCH}_{2} \mathrm{CH}_{2} \mathrm{CH}_{2} \mathrm{~N}\right.$ ), 70.1 (C-5GalN), 70.9 (C-5Gal, C-3Fuc), 71.4 (C-3GalN), 72.1 (C-4Fuc), 73.0 (C-2Fuc), 73.2 (C-3Gal), $73.5\left(\mathrm{CH}_{2}-\mathrm{Ph}\right), 77.4$ (C-2Gal), 93.5 (C-1GalN), 99.1 (C-1Fuc), 103.3 (C-1Gal), 127.7-137.9(6Ph), 164.9, 165.9, 166.0, 166.1, 166.4 (5 COPh). 14: ${ }^{1} \mathrm{H}$ NMR $\left(400 \mathrm{MHz}, \mathrm{CDCl}_{3}\right): 1.21\left(\mathrm{~d}, 3 \mathrm{H}, J=6.5 \mathrm{~Hz} ; \mathrm{CH}_{3}-\mathrm{Fuc}\right), 1.95-2.00\left(\mathrm{~m}, 2 \mathrm{H}, \mathrm{OCH}_{2} \mathrm{CH}_{2} \mathrm{CH}_{2} \mathrm{~N}\right)$, $3.33(\mathrm{dd}, 1 \mathrm{H}, J=4.1 ; 12.2 \mathrm{~Hz} ; \mathrm{H}-6 \mathrm{aGalN}), 3.39(\mathrm{dd}, 1 \mathrm{H}, J=4.5 ; 12.2 \mathrm{~Hz} ; \mathrm{H}-6 \mathrm{bGalN}), 3.44-$ $3.60\left(\mathrm{~m}, 2 \mathrm{H}, \mathrm{OCH}_{2} \mathrm{CH}_{2} \mathrm{CH}_{2} \mathrm{~N}\right), 3.79-3.85\left(\mathrm{~m}, 1 \mathrm{H}, \mathrm{OC} \underline{\mathrm{H}} \mathrm{HCH}_{2} \mathrm{CH}_{2} \mathrm{~N}\right), 4.00-4.20(\mathrm{~m}, 8 \mathrm{H}$, $\left.\mathrm{OCH} \mathrm{HCH}_{2} \mathrm{CH}_{2} \mathrm{~N}, \mathrm{H}-2 \mathrm{GalN}, \mathrm{H}-2 \mathrm{Gal}, \mathrm{H}-2 \mathrm{Fuc}, \mathrm{H}-5 \mathrm{Gal}, \mathrm{H}-5 \mathrm{GalN}, \mathrm{CH}_{2}-\mathrm{Ph}\right), 4.44(\mathrm{~d}, 1 \mathrm{H}$, $J=3.0 \mathrm{~Hz} ; \mathrm{H}-4 \mathrm{GalN}), 4.46(\mathrm{~d}, 1 \mathrm{H}, J=2.9 \mathrm{~Hz} ; \mathrm{H}-4 \mathrm{Gal}), 4.66-4.80(\mathrm{~m}, \overline{4 \mathrm{H}}, \mathrm{H}-1 \mathrm{Gal}, \mathrm{H}-5 \mathrm{Fuc}$, H-6Gal), $5.14(\mathrm{~d}, 1 \mathrm{H}, J=3.6 \mathrm{~Hz} ; \mathrm{H}-1 \mathrm{GalN}), 5.42(\mathrm{dd}, 1 \mathrm{H}, J=2.9 ; 10.1 \mathrm{~Hz} ; \mathrm{H}-3 \mathrm{Gal}), 5.50$ (dd, $1 \mathrm{H}, J=2.9 ; 11.0 \mathrm{~Hz} ; \mathrm{H}-3 \mathrm{GalN}), 5.54(\mathrm{~d}, 1 \mathrm{H}, J=3.5 \mathrm{~Hz} ; \mathrm{H}-1 \mathrm{Fuc}), 5.60-5.67$ (m, 2H, H-4Fuc, H-3Fuc), 6.77-8.20 (m, 30H, 6Ph). $\left.{ }^{13} \mathrm{C} \mathrm{NMR} \mathrm{(100} \mathrm{MHz,} \mathrm{CDCl}\right)$ ): 15.9 (C-6Fuc), 28.5 $\left(\mathrm{OCH}_{2} \mathrm{CH}_{2} \mathrm{CH}_{2} \mathrm{~N}\right), 37.8\left(\mathrm{OCH}_{2} \mathrm{CH}_{2} \mathrm{CH}_{2} \mathrm{~N}\right), 58.3(\mathrm{C}-2 \mathrm{GalN}), 62.2(\mathrm{C}-6 \mathrm{Gal}), 63.0(\mathrm{C}-6 \mathrm{GalN})$, 65.6 (C-5Fuc), $67.7\left(\mathrm{OCH}_{2} \mathrm{CH}_{2} \mathrm{CH}_{2} \mathrm{~N}\right), 68.9$ (C-4GalN), 69.7 (C-5GalN), 70.3 (C-3Fuc), 71.9 (C-3GalN), 72.1 (C-4Fuc), 72.2 ( $\left.\mathrm{CH}_{2}-\mathrm{Ph}\right), 72.3$ (C-5Gal), 72.6 (C-2Fuc), 74.5 (C-4Gal), 75.8 (C3Gal), 97.2 (C-1Fuc), 99.3 (C-1GalN), 102.3 (C-1Gal), 127.6-133.9 (6Ph), 165.5, 165.9 (2 COPh). HRMS (ESI) $m / z$ : found $\left[\mathrm{M}+\mathrm{NH}_{4}\right]^{+} 1294.4324, \mathrm{C}_{65} \mathrm{H}_{63} \mathrm{~F}_{3} \mathrm{~N}_{4} \mathrm{O}_{20}$ calcd for $\left[\mathrm{M}+\mathrm{NH}_{4}\right]^{+}$ 1294.4326.

B: A carefully dried mixture of donor $10(84.8 \mathrm{mg}, 0.14 \mathrm{mmol}, 1.2 \mathrm{eq}$.$) and disaccharide$ acceptor 9 (118 mg, $0.12 \mathrm{mmol})$ was dissolved in $\mathrm{CH}_{2} \mathrm{Cl}_{2}(2 \mathrm{~mL})$ and molecular sieves $4 \AA$ ( $200 \mathrm{mg})$ were added. After $10 \mathrm{~min}$, MeOTf $\left(98.7 \mu \mathrm{L}, 0.9 \mathrm{mmol}, 7.5\right.$ eq.) and $\mathrm{Me}_{2} \mathrm{~S}_{2}$ $(79.7 \mu \mathrm{L}, 0.9 \mathrm{mmol}, 7.5 \mathrm{eq}$.) were added. The mixture was stirred for $2 \mathrm{~h}$ at room temperature and quenched with one drop of $\mathrm{Et}_{3} \mathrm{~N}$. The mixture was filtered through a Celite pad with $\mathrm{CH}_{2} \mathrm{Cl}_{2}$ and the filtrate was washed with sat. aq. $\mathrm{NaHCO}_{3}$. The organic phase was separated, and the solvent was evaporated in vacuo. The residue was purified by flash chromatography (toluene:ethyl acetate $0 \rightarrow 20 \%$ ), giving $137.6 \mathrm{mg}(81 \%)$ of trisaccharide 15 (contaminated by traces of $(1 \rightarrow 4)$-glycosylation product). To a solution of the purified compound in Py $(2 \mathrm{~mL})$ was added $40 \%$ aq. HF $(333 \mu \mathrm{L})$. After $10 \mathrm{~min}$, the mixture was diluted with EtOAc, washed with $1 \mathrm{M}$ aq. $\mathrm{HCl}$, sat. aq. $\mathrm{NaHCO}_{3}$, the combined organic phase was dried by filtration through $\mathrm{Na}_{2} \mathrm{SO}_{4}$, and concentrated in vacuo. The residue was purified by flash chromatography (toluene:ethyl acetate $20 \rightarrow 50 \%)$, giving $117.9 \mathrm{mg}(77 \%$; summary yield $62 \%$ ) of trisaccharide 13 . All spectral characteristics w completely identical to the compound $\mathbf{1 3}$ obtained by method $\mathbf{A}$.

3.2.5. 3-Aminopropyl 2-Acetamido-2-Deoxy- $\alpha$-D-Galactopyranosyl-(l-3)-[( $\alpha$-L-Fucopy-

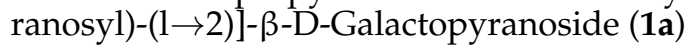

To a solution of the trisaccharide $\mathbf{1 3}(83.8 \mathrm{mg}, 66 \mu \mathrm{mol})$ in EtOAc $(2 \mathrm{~mL})$ and $\mathrm{MeOH}$ $(1 \mathrm{~mL})$ were added $1 \mathrm{M} \mathrm{HCl}(50 \mu \mathrm{L})$ and $\mathrm{Pd}(\mathrm{OH})_{2} / \mathrm{C}(100 \mathrm{mg})$ after the flask was filled with hydrogen. The reaction mixture was stirred for $3 \mathrm{~h}$ at RT. Then, the reaction mixture was filtered on a glass filter through a Celite pad and concentrated in vacuo. The crude material was dissolved in $\mathrm{CHCl}_{3}: \mathrm{MeOH}\left(2 \mathrm{~mL}\right.$ in ratio 1:1), then $\mathrm{Et}_{3} \mathrm{~N}(27 \mu \mathrm{L}, 0.19 \mathrm{mmol})$ and $\mathrm{Ac}_{2} \mathrm{O}(12 \mu \mathrm{L}, 0.13 \mathrm{mmol})$ were added. After completing the reaction, the mixture was concentrated in vacuo and purified by flash chromatography $\left(\mathrm{CHCl}_{3}: \mathrm{MeOH} 0 \rightarrow 10 \%\right)$, 
giving $57.3 \mathrm{mg}(68 \%)$ of trisaccharide 16 . One $\mathrm{M} \mathrm{MeONa}(100 \mu \mathrm{L})$ was added to a solution of the purified compound in $\mathrm{MeOH}: \mathrm{CH}_{2} \mathrm{Cl}_{2}(0.8 \mathrm{~mL}$ in ratio 3:1). The mixture was left for $2 \mathrm{~h}$, then $4 \mathrm{M} \mathrm{NaOH}(50 \mu \mathrm{L})$ was added and left overnight. The base was neutralized by $1 \mathrm{M}$ aq. $\mathrm{HCl}$ and the resulting solution was concentrated. The residue was purified by gel-permeation chromatography (TSK HW-40 (S), $0.1 \mathrm{M} \mathrm{AcOH}$ ) giving $19.4 \mathrm{mg}$ (73\%) of trisaccharide 1a. All NMR and HRMS data corresponded to the literature data [9].

3.2.6. 3-(21-Biotinamino-4,7,10,13,16,19-Hexaoxagenicaminoamino)-Propyl

2-Acetamido-2-Deoxy- $\alpha$-D-Galactopyranosyl- $(1 \rightarrow 3)-[(\alpha-L-F u c o p y r a n o s y l)-(1 \rightarrow 2)]-\beta-D-$

Galactopyranoside (1b)

To a solution of trisaccharide $1 \mathrm{a}(0.5 \mathrm{mg}, 0.85 \mu \mathrm{mol})$ in DMF $(100 \mu \mathrm{L})$ was added a $0.0062 \mathrm{M}$ solution of biotin-activated ester $17(20 \mu \mathrm{L}, 1.28 \mu \mathrm{mol}, 1.5$ eq. $)$ and $\mathrm{Et}_{3} \mathrm{~N}$ $(15 \mu \mathrm{L}, 0.1 \mathrm{mmol})$. After $20 \mathrm{~min}$, the mixture was concentrated in vacuo, after which the residue was purified by gel-permeation chromatography (G-15, $0.1 \mathrm{M} \mathrm{AcOH})$ to give $0.85 \mathrm{mg}(87 \%)$ biotinylated conjugate $\mathbf{1 b} .{ }^{1} \mathrm{H} \mathrm{NMR}\left(600 \mathrm{MHz}, \mathrm{D}_{2} \mathrm{O}\right.$, characteristic signals): oligosaccharide fragment: $1.24\left(\mathrm{~d}, 3 \mathrm{H}, J=6.6 \mathrm{~Hz} ; \mathrm{CH}_{3}-\mathrm{Fuc}\right), 1.93(\mathrm{~s}, 1 \mathrm{H}, \mathrm{NH} \underline{\mathrm{Ac}}), 4.55(\mathrm{~d}$, $\overline{1 \mathrm{H}}, J=7.8 \mathrm{~Hz}$; H-1Gal), 5.20 (d, 1H, $J=3.7 \mathrm{~Hz} ; \mathrm{H}-1 \mathrm{GalN}), 5.32$ (d, 1H, $J=3.8 \mathrm{~Hz} ; \mathrm{H}-1 \mathrm{Fuc}$ ); biotin fragment: 1.42-1.78 (m, 6H, H-b, H-c, H-d), 2.30 (t, 2H, J = 7.3 Hz; H-a), 2.81 (d, $\left.\overline{1 \mathrm{H}, J}=13.0 \mathrm{~Hz} ; \mathrm{H}-\mathrm{h}^{\prime}\right), 3.02(\mathrm{dd}, 1 \mathrm{H}, \mathrm{J}=5 \mathrm{~Hz} ; 13.1 \mathrm{~Hz} ; \mathrm{H}-\mathrm{h}), 3.34-3.38$ (m, 1H, H-e), 4.63 (dd, 1H, J = 5.0 Hz; 7.9 Hz; H-g); linker: 1.83-1.89 (m, 2H, OCH $\left.\mathrm{CH}_{2} \mathrm{CH}_{2} \mathrm{~N}\right), 2.54(\mathrm{t}, 2 \mathrm{H}$, $\left.J=6.1 \mathrm{~Hz} ; \mathrm{OCH}_{2} \mathrm{CH}_{2} \mathrm{CH}_{2} \mathrm{~N}\right) \cdot{ }^{13} \mathrm{C}$ NMR characteristic signals derived from $\left({ }^{1} \mathrm{H},{ }^{13} \mathrm{C}\right) \mathrm{HSQC}$ spectrum $\left(\mathrm{D}_{2} \mathrm{O}\right)$ : oligosaccharide fragment: $16.7\left(\mathrm{CH}_{3}-\mathrm{Fuc}\right), 26.4(\mathrm{NH} \underline{A c}), 92.7(\mathrm{C}-1 \mathrm{GalN})$, 98.8 (C-1Fuc), 102.7 (C-1Gal); biotin fragment: 26.4 (C-c), 29.1 (C-b, C-d), 36.7 (C-a), 56.8 (C-e), 61.6 (C-g); linker: $29.8\left(\mathrm{OCH}_{2} \mathrm{CH}_{2} \mathrm{CH}_{2} \mathrm{~N}\right), 37.4\left(\mathrm{OCH}_{2} \mathrm{CH}_{2} \mathrm{CH}_{2} \mathrm{~N}\right)$. HRMS (ESI) $m / z$ : found $[\mathrm{M}+\mathrm{Na}]^{+} 1170.5197, \mathrm{C}_{48} \mathrm{H}_{85} \mathrm{~N}_{5} \mathrm{O}_{24} \mathrm{~S}$ calcd for $[\mathrm{M}+\mathrm{Na}]^{+} 1170.5178$.

\section{Conclusions}

The synthesis of spacered A trisaccharide derivatives $\mathbf{1 a}$ and $\mathbf{1} \mathbf{b}$ was performed using a 2-azido-2-deoxy-selenogalactoside glycosyl donor bearing stereo-controlling 3-O-benzoyl and 4,6-O-(di-tert-butylsilylene)-protecting groups, showing once again the efficacy of this $\alpha$-glycosylation agent for the assembly of even vicinally branched oligosaccharide chains. At the same time, we observed rather poor applicability of donor $\mathbf{1 0}$ for the regioselective glycosylation of diolic acceptor $\mathbf{8}$. Obtained trisaccharide $\mathbf{1 b}$ is being used in the coating of magnetic nanobeads for glycobiological applications to be described in due course.

Supplementary Materials: The following are available online. Copies of NMR spectra of compounds $4,6-9,13,14,1 a$, and $1 b$.

Author Contributions: D.V.Y. and N.E.N. conceived the project and designed the experiments. E.D.K. performed the experiments. E.D.K., D.V.Y., and N.E.N. interpreted the data and wrote the manuscript. N.E.N. acquired funding. All authors have read and agreed to the published version of the manuscript.

Funding: The work was supported by the Ministry of Science and Higher Education of Russia (Grant Agreement No. 075-15-2020-792).

Institutional Review Board Statement: Not applicable.

Informed Consent Statement: Not applicable.

Data Availability Statement: Data are available from the corresponding author.

Acknowledgments: The authors thank N.E. Ustyuzhanina and D.Z. Vinnitsky for their contribution to the preliminary experiments to this work, D.A. Argunov for recording the NMR spectra and helping with interpreting the data, and also A.I. Tokatly for reading this manuscript and its critical discussion.

Conflicts of Interest: The authors declare no conflict of interest. 
Sample Availability: Samples of described compounds are available from the corresponding author.

\section{References}

1. Morgan, W.T.J.; Watkins, W.M. Genetic and Biochemical Aspects of Human Blood-Group A-, B-, H-, Le-a- and Le-b-Specificity. Br. Med. Bull. 1969, 25, 30-34. [CrossRef] [PubMed]

2. Rege, V.P.; Painter, T.J.; Watkins, W.M.; Morgan, W.T.J. Isolation of Serologically Active Fucose-Containing Oligosaccharides from Human Blood-Group H Substance. Nature 1964, 203, 360-363. [CrossRef] [PubMed]

3. Bovin, N.V.; Zurabyan, S.É.; Khorlin, A.Y. Stereoselectivity in Glycosylation by Means of 2-Azido-2-Desoxy-D-Galactopyranose Derivatives and the Synthesis of the Determinative Oligosaccharide of Blood Group A, Type 1. Russ. Chem. Bull. 1982, 31, 1023-1030. [CrossRef]

4. Åberg, P.-M.; Blomberg, L.; Lönn, H.; Norberg, T. Glycosylation with Thioglycosides Activated by Dimethyl(Methylthio)Sulfonium Tetrafluoroborate: Synthesis of Two Trisaccharide Glycosides Corresponding to the Blood Group A and B Determinants. Glycoconj. J. 1990, 7, 201-205. [CrossRef]

5. EIu, K.; Bovina, N.V. Synthesis of spaced trisaccharides with blood group A and B specificity, their fragments, and structural analogs. Bioorg. Khim. 1992, 18, 283-298.

6. Khatuntseva, E.A.; Tsvetkov, Y.E.; Grachev, A.A.; Nifant'ev, N.E. Synthesis of Aminoethyl Glycosides of Type 2 Chain A Tetrasaccharide and Related Trisaccharides. Russ. J. Org. Chem. 2005, 41, 1814-1823. [CrossRef]

7. Meloncelli, P.J.; Lowary, T.L. Synthesis of ABO Histo-Blood Group Type I and II Antigens. Carbohydr. Res. 2010, 345, 2305-2322. [CrossRef] [PubMed]

8. Hara, A.; Imamura, A.; Ando, H.; Ishida, H.; Kiso, M. A New Chemical Approach to Human ABO Histo-Blood Group Type 2 Antigens. Molecules 2014, 19, 414-437. [CrossRef]

9. Karki, G.; Mishra, V.N.; Mandal, P.K. An Expeditious Synthesis of Blood-Group Antigens, ABO Histo-Blood Group Type II Antigens and Xenoantigen Oligosaccharides with Amino Type Spacer-arms. Glycoconj. J. 2016, 33, 63-78. [CrossRef] [PubMed]

10. Kunetskiy, R.A.; Pazynina, G.V.; Ivanov, I.A.; Bovin, N.V. Synthesis of Blood Group A and B (Type 2) Tetrasaccharides. A Strategy with Fucosylation at the Last Stage. Carbohydr. Res. 2020, 498, 108192. [CrossRef]

11. West, L.J.; Pollock-Barziv, S.M.; Dipchand, A.I.; Lee, K.J.; Cardella, C.J.; Benson, L.N.; Rebeyka, I.M.; Coles, J.G. ABO-Incompatible Heart Transplantation in Infants. N. Engl. J. Med. 2001, 344, 793-800. [CrossRef]

12. Yamamoto, F. Review: ABO Blood Group System-ABH Oligosaccharide Antigens, Anti-A and Anti-B, A and B Glycosyltransferases, and ABO Genes. Immunohematology 2004, 20, 3-22. [CrossRef]

13. Bentall, A.; Jeyakanthan, M.; Braitch, M.; Cairo, C.W.; Lowary, T.L.; Maier, S.; Halpin, A.; Motyka, B.; Zou, L.; West, L.J.; et al. Characterization of ABH-Subtype Donor-Specific Antibodies in ABO-A-Incompatible Kidney Transplantation. Am. J. Transplant. 2021. [CrossRef]

14. Casset, F.; Peters, T.; Etzler, M.; Korchagina, E.; Nifant'ev, N.; Pérez, S.; Imberty, A. Conformational Analysis of Blood Group A Trisaccharide in Solution and in the Binding Site of Dolichos Biflorus Lectin Using Transient and Transferred Nuclear Overhauser Enhancement (NOE) and Rotating-Frame NOE Experiments. Eur. J. Biochem. 1996, 239, 710-719. [CrossRef]

15. Iurisci, I.; Cumashi, A.; Sherman, A.A.; Tsvetkov, Y.E.; Tinari, N.; Piccolo, E.; D'egidio, M.; Adamo, V.; Natoli, C.; Rabinovich, G.A.; et al. Synthetic Inhibitors of Galectin-1 and -3 Selectively Modulate Homotypic Cell Aggregation and Tumor Cell Apoptosis. Anticancer Res. 2009, 29, 403-410.

16. Quintana, J.I.; Delgado, S.; Núñez-Franco, R.; Cañada, F.J.; Jiménez-Osés, G.; Jiménez-Barbero, J.; Ardá, A. Galectin-4 N-Terminal Domain: Binding Preferences Toward A and B Antigens With Different Peripheral Core Presentations. Front. Chem. 2021, 9, 193. [CrossRef] [PubMed]

17. Lipkind, G.M.; Shashkov, A.S.; Nifant'ev, N.E.; Kochetkov, N.K. Computer-Assisted Analysis of the Structure of Regular Branched Polysaccharides Containing 2,3-Disubstituted Rhamnopyranose and Mannopyranose Residues on the Basis of ${ }^{13} \mathrm{C}$ NMR Data. Carbohydr. Res. 1992, 237, 11-22. [CrossRef]

18. Gerbst, A.G.; Ustuzhanina, N.E.; Grachev, A.A.; Khatuntseva, E.A.; Tsvetkov, D.E.; Whitfield, D.M.; Berces, A.; Nifantiev, N.E. Synthesis, NMR, and Conformational Studies of Fucoidan Fragments. III. Effect of Benzoyl Group at O-3 on Stereoselectivity of Glycosylation by 3-O- and 3,4-Di-O-Benzoylated 2-O-Benzylfucosyl Bromides. J. Carbohydr. Chem. 2001, 20, 821-831. [CrossRef]

19. Khatuntseva, E.A.; Ustuzhanina, N.E.; Zatonskii, G.V.; Shashkov, A.S.; Usov, A.I.; Nifantiev, N.E. The Synthesis and NMR and Conformational Studies of Fucoidan Fragments: I Synthesis, NMR and conformational studies of fucoidan fragments. Part 1. Desulfated 2,3- and 3,4-branched trisaccharide fragments and costituing disaccharides. J. Carbohydr. Chem. 2000, 19, 1151-1173. [CrossRef]

20. Kazakova, E.D.; Yashunsky, D.V.; Krylov, V.B.; Bouchara, J.-P.; Cornet, M.; Valsecchi, I.; Fontaine, T.; Latgé, J.-P.; Nifantiev, N.E. Biotinylated Oligo- $\alpha-(1 \rightarrow 4)$-d-Galactosamines and Their N-Acetylated Derivatives: $\alpha$-Stereoselective Synthesis and Immunology Application. J. Am. Chem. Soc. 2020, 142, 1175-1179. [CrossRef] [PubMed]

21. Imamura, A.; Matsuzawa, N.; Sakai, S.; Udagawa, T.; Nakashima, S.; Ando, H.; Ishida, H.; Kiso, M. The Origin of High Stereoselectivity in Di-Tert-Butylsilylene-Directed $\alpha$-Galactosylation. J. Org. Chem. 2016, 81, 9086-9104. [CrossRef]

22. Komarova, B.S.; Tsvetkov, Y.E.; Nifantiev, N.E. Design of $\alpha$-Selective Glycopyranosyl Donors Relying on Remote Anchimeric Assistance. Chem. Rec. 2016, 16, 488-506. [CrossRef] 
23. Kazakova, E.D.; Yashunsky, D.V.; Khatuntseva, E.A.; Nifantiev, N.E. Azidophenylselenylation of Glycals towards 2-Azido-2Deoxy-Selenoglycosides and Their Application in Oligosaccharide Synthesis. Pure Appl. Chem. 2020, 92, 1047-1056. [CrossRef]

24. Nigudkar, S.S.; Demchenko, A.V. Stereocontrolled 1,2- Cis Glycosylation as the Driving Force of Progress in Synthetic Carbohydrate Chemistry. Chem. Sci. 2015, 6, 2687-2704. [CrossRef]

25. Milner, S.J.; Carrick, C.T.; Kerr, K.G.; Snelling, A.M.; Thomas, G.H.; Duhme-Klair, A.-K.; Routledge, A. Probing Bacterial Uptake of Glycosylated Ciprofloxacin Conjugates. ChemBioChem 2014, 15, 466-471. [CrossRef]

26. Kochetkov, N.K.; Nifant'ev, N.E.; Backinowsky, L.V. Synthesis of the Capsular Polysaccharide of Streptococcus Pneumoniae Type 14. Tetrahedron 1987, 43, 3109-3121. [CrossRef]

27. Mironov, Y.V.; Sherman, A.A.; Nifantiev, N.E. Homogeneous Azidophenylselenylation of Glycals Using TMSN3-Ph2Se2PhI(OAc)2. Tetrahedron Lett. 2004, 45, 9107-9110. [CrossRef]

28. Khatuntseva, E.A.; Sherman, A.A.; Tsvetkov, Y.E.; Nifantiev, N.E. Phenyl 2-Azido-2-Deoxy-1-Selenogalactosides: A Single Type of Glycosyl Donor for the Highly Stereoselective Synthesis of $\alpha$ - and $\beta$-2-Azido-2-Deoxy-d-Galactopyranosides. Tetrahedron Lett. 2016, 57, 708-711. [CrossRef]

29. Hagen, B.; Ali, S.; Overkleeft, H.S.; van der Marel, G.A.; Codée, J.D.C. Mapping the Reactivity and Selectivity of 2-Azidofucosyl Donors for the Assembly of N-Acetylfucosamine-Containing Bacterial Oligosaccharides. J. Org. Chem. 2017, 82, 848-868. [CrossRef] [PubMed]

30. Zhang, Y.; Gómez-Redondo, M.; Jiménez-Osés, G.; Arda, A.; Overkleeft, H.S.; van der Marel, G.A.; Jiménez-Barbero, J.; Codée, J.D.C. Synthesis and Structural Analysis of Aspergillus Fumigatus Galactosaminogalactans Featuring $\alpha$-Galactose, $\alpha$-Galactosamine and $\alpha-\mathrm{N}$-Acetyl Galactosamine Linkages. Angew. Chem. Int. Ed. 2020, 59, 12746-12750. [CrossRef] [PubMed]

31. Li, C.; Liu, G.; Du, W.; Zhang, Y.; Xing, G. A Novel O-Fucosylation Strategy Preactivated by (p-Tol)2SO/Tf2O and Its Application for the Synthesis of Lewis Blood Group Antigen Lewisa. Tetrahedron Lett. 2017, 58, 2109-2112. [CrossRef]

32. Vigo, E.A.D.; Stortz, C.A.; Marino, C. Regioselectivity of Glycosylation Reactions of Galactose Acceptors: An Experimental and Theoretical Study. Beilstein J. Org. Chem. 2019, 15, 2982-2989. [CrossRef] [PubMed]

33. Tsvetkov, Y.E.; Burg-Roderfeld, M.; Loers, G.; Ardá, A.; Sukhova, E.V.; Khatuntseva, E.A.; Grachev, A.A.; Chizhov, A.O.; Siebert, H.-C.; Schachner, M.; et al. Synthesis and Molecular Recognition Studies of the HNK-1 Trisaccharide and Related Oligosaccharides. The Specificity of Monoclonal Anti-HNK-1 Antibodies as Assessed by Surface Plasmon Resonance and STD NMR. J. Am. Chem. Soc. 2012, 134, 426-435. [CrossRef] [PubMed] 